\title{
Global Dynamics and Optimal Control of a Viral Infection Model with Generic Nonlinear Infection Rate
}

\author{
Chenquan Gan, ${ }^{1,2}$ Maobin Yang, ${ }^{1}$ Zufan Zhang, ${ }^{1}$ and Wanping Liu ${ }^{1,2}$ \\ ${ }^{1}$ School of Communication and Information Engineering, Chongqing University of Posts and Telecommunications, \\ Chongqing 400065, China \\ ${ }^{2}$ College of Computer Science and Engineering, Chongqing University of Technology, Chongqing 400054, China
}

Correspondence should be addressed to Wanping Liu; lwphe@163.com

Received 16 October 2016; Revised 5 December 2016; Accepted 25 December 2016; Published 13 February 2017

Academic Editor: Jose R. C. Piqueira

Copyright (C) 2017 Chenquan Gan et al. This is an open access article distributed under the Creative Commons Attribution License, which permits unrestricted use, distribution, and reproduction in any medium, provided the original work is properly cited.

\begin{abstract}
This paper is devoted to exploring the combined impact of a generic nonlinear infection rate and infected removable storage media on viral spread. For that purpose, a novel dynamical model with an external compartment is proposed, and the explanations of the main model assumptions (especially the generic nonlinear infection rate) are also examined. The existence and global stability of the unique equilibrium of the model are fully investigated, from which it can be seen that computer virus would persist. On this basis, a next-best approach to controlling the level of infected computers is suggested, and the theoretical analysis of optimal control of the model is also performed. Additionally, some numerical examples are given to illustrate the main results.
\end{abstract}

\section{Introduction}

In the wake of developments in computer and network technologies, computer virus has become more capable of conquering computer system. In the meantime, the study of fighting against computer virus has in the past few decades been paid more attention. In reality, there is no doubt that antivirus software and firewall are the most effective prevention measures. However, they are incompetent to inhibit computer virus diffusion over the Internet [1]. To deal with this problem, a wide variety of mathematical models have been widely studied (for the related references, see, e.g., [215]).

The infection rate is an important and essential system parameter in computer virus propagation models. However, the dominating majority of previous models assume a bilinear incidence rate (for the related references, see, e.g., [16-20]) or a nonlinear increasing incidence rate (for the related references, see, e.g., [21]). The former assumption is suitable for the case where the proportion of infected computers is small. The latter assumption neglects the fact that, due to active protection measures taken during viral spread, the infection rate would be decreasing, while the infected computers may be increasing. In order to depict the case where the infection rate could be decreasing with the infected computers and inspired by the previous work (e.g., $[22,23])$, the proposed model of this paper adopts a nonlinear function $\sigma(I)=\beta I / f(I)$, where $\beta>0$ and function $f \in C^{2}[0,+\infty)$ with $f^{\prime} \geq 0, f^{\prime \prime}<0$, and $f(0)=1$ (see also the model assumption (A3) in the next section).

External computers (i.e., computers outside the Internet) and infected removable storage media play an important role in viral spread (for the related references, see, e.g., $[24,25])$. In [24], the impact of infected removable storage media is considered, but the influence of external computers is insufficient. In [25], a dynamical model, in which all external computers are regarded as a separate compartment, was proposed. Unfortunately, this model ignores the effects of generic nonlinear infection rate and infected removable storage media. Consequently, it is necessary to consider the combined impact of a generic nonlinear infection rate and infected removable storage media on viral spread.

In addition, optimal control theory is often applied to control virus prevalence (for the related references, see, e.g., $[14,26-28])$. In [14, 27], a susceptible-latent-breaking-outside-susceptible (SLBOS) model and a susceptiblelatent-breaking-susceptible (SLBS) model were studied, 
respectively. References $[26,28]$ considered a susceptibleinfected-recovered-susceptible (SIRS) model in a fully connected network and a complex network, respectively. To our knowledge, there is no susceptible-infected-externalsusceptible (SIES) model that has been examined by applying optimal control theory.

Combining the above discussions, a novel SIES model with two kinds of incidence rates, which are caused by infected computers and infected removable storage media, is established in this paper. A systematic analysis of the proposed model shows that the unique (viral) equilibrium is globally asymptotically stable. This result indicates that any effort in eradicating computer virus cannot succeed. In this regard, theoretical analysis of a next-best approach and optimal control of the model is performed. Numerical analysis of the model is also included.

The remaining materials of this paper are organized as follows: Section 2 formulates the model. Section 3 considers the viral equilibrium and its global stability. In Section 4, a theoretical analysis of optimal control of the model is performed. Finally, Section 5 concludes the contributions of this work and points out some further works that are worth doing.

\section{Model Characterization}

In this paper, the proposed model consists of three compartments (see (i)-(iii)), and the assumptions of it are also made (see (A1)-(A8)):

(i) S-compartment: the set of all S-computers (susceptible internal computers, i.e., computers in the Internet)

(ii) $I$-compartment: the set of all $I$-computers (infected internal computers)

(iii) E-compartment: the set of all $E$-computers (external computers, i.e., computers outside the Internet)

(A1) Every computer is out of use with probability per unit time $\mu>0$

(A2) Every $S$ - or $I$-computer leaves the Internet with probability per unit time $\gamma_{1}>0$

(A3) Due to possible communication with $I$-computers over the Internet, every $S$-computer is infected with probability per unit time $\sigma(I)=\beta I / f(I)$, where $\beta>0$ and function $f \in C^{2}[0,+\infty)$ with $f^{\prime} \geq 0, f^{\prime \prime}<0$, and $f(0)=1$

(A4) Due to possible effect of infected removable storage media, every $S$-computer is infected with probability per unit time $\theta \geq 0$

(A5) Due to treatment, every $I$-computer is cured with probability per unit time $\gamma_{2}>0$

(A6) The rate of all newly accessed $E$-computers is $\delta>0$

(A7) Every E-computer is either susceptible or infected when it enters the Internet

(A8) Every susceptible (or infected) $E$-computer enters the Internet with probability per unit time $\eta_{2}>0$ (or $\eta_{1}>$ 0)

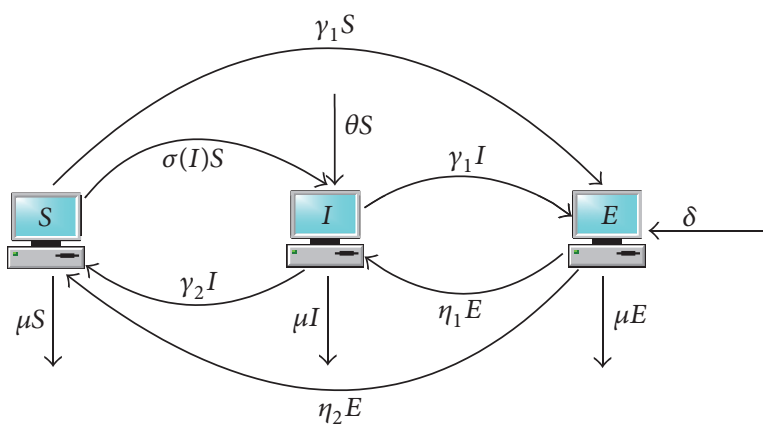

FIGURE 1: The transfer diagram of the proposed model.

For convenience, let $S, I$, and $E$ represent the average number of computers in $S$-compartment, $I$-compartment, and $E$-compartment at time $t$, respectively. Collecting the foregoing hypotheses, the proposed model can be depicted by Figure 1 or the differential system

$$
\begin{aligned}
& \dot{S}=\gamma_{2} I+\eta_{2} E-\mu S-\sigma(I) S-\gamma_{1} S-\theta S, \\
& \dot{I}=\sigma(I) S+\theta S-\mu I-\gamma_{1} I-\gamma_{2} I+\eta_{1} E, \\
& \dot{E}=\delta+\gamma_{1} S+\gamma_{1} I-\mu E-\eta_{1} E-\eta_{2} E,
\end{aligned}
$$

with initial condition $(S(0), I(0), E(0)) \in R_{+}^{3}$.

\section{Model Analysis}

Let $N=S+I+E$. System (1) can be rewritten as

$$
\begin{aligned}
\dot{N}= & \delta-\mu N, \\
\dot{I}= & \sigma(I)(N-I-E)+\theta(N-I-E)-\mu I-\gamma_{1} I \\
& -\gamma_{2} I+\eta_{1} E, \\
\dot{E}= & \delta+\gamma_{1} N-\gamma_{1} E-\mu E-\eta_{1} E-\eta_{2} E,
\end{aligned}
$$

with initial condition $(N(0), I(0), E(0)) \in R_{+}^{3}$.

Let $N^{*}=\delta / \mu$ and $E^{*}=\delta\left(\mu+\gamma_{1}\right) / \mu\left(\mu+\gamma_{1}+\eta_{1}+\eta_{2}\right)$. Solving the first and third equations of system (2), we get $\lim _{t \rightarrow+\infty} N(t)=N^{*}$ and $\lim _{t \rightarrow+\infty} E(t)=E^{*}$. System (2) can be reduced to the limiting system [29]

$$
\dot{I}=\sigma(I)(A-I)-\left(\mu+\gamma_{1}+\gamma_{2}+\theta\right) I+B,
$$

where $A=N^{*}-E^{*}=\delta\left(\eta_{1}+\eta_{2}\right) / \mu\left(\mu+\gamma_{1}+\eta_{1}+\eta_{2}\right)>0$ and $B=\eta_{1} E^{*}+\theta A>0$.

Clearly, system (3) has no virus-free equilibrium. Thus, this section mainly addresses the existence and global stability of viral equilibrium of system (3) with respect to positively invariant region: $\Omega=\left\{I \mid 0 \leq I \leq N^{*}\right\}$.

\subsection{Equilibrium}

Theorem 1. System (3) has a unique viral equilibrium $I^{*}$, where $I^{*}$ is the unique root in $\left(0, N^{*}\right)$ of the equation

$$
F(x)=\sigma(x)(A-x)-\left(\mu+\gamma_{1}+\gamma_{2}+\theta\right) x+B=0 .
$$


Proof. If $I^{*}$ is a viral equilibrium of system (3), then it satisfies (4). Now, it suffices to show that (4) has a unique root in $\left(0, N^{*}\right)$. As $\sigma(x)=\beta x / f(x)$ and $\sigma^{\prime}(x)=\beta((f(x)-$ $\left.\left.x f^{\prime}(x)\right) / f^{2}(x)\right)$, we proceed by treating two possibilities.

Case 1. $f(x)-x f^{\prime}(x) \leq 0$; namely, $\sigma^{\prime}(x) \leq 0$. Note that

$$
\begin{aligned}
F(0)= & B>0, \\
F(A)= & B-\left(\mu+\gamma_{1}+\gamma_{2}+\theta\right) A \\
= & -\frac{\delta\left(\eta_{2}\left(\mu+\gamma_{1}+\gamma_{2}\right)+\gamma_{2} \eta_{1}\right)}{\mu\left(\mu+\gamma_{1}+\eta_{1}+\eta_{2}\right)}<0, \\
F^{\prime}(x)= & \beta \frac{(A-2 x) f(x)-(A-x) x f^{\prime}(x)}{f^{2}(x)} \\
& -\left(\mu+\gamma_{1}+\gamma_{2}+\theta\right) \\
= & \beta \frac{A\left(f(x)-x f^{\prime}(x)\right)-x\left[2 f(x)-x f^{\prime}(x)\right]}{f^{2}(x)} \\
& -\left(\mu+\gamma_{1}+\gamma_{2}+\theta\right) .
\end{aligned}
$$

Let $G(x)=2 f(x)-x f^{\prime}(x)$; then $G(0)=2 f(0)>0 . f(x)-$ $x f^{\prime}(x) \leq 0, f^{\prime} \geq 0 ; f^{\prime \prime}<0$ implies that $G^{\prime}(x)=f^{\prime}(x)-$ $x f^{\prime \prime}(x)>0$. Hence, $G(x)>0 ; F^{\prime}(x)<0$ implies that $F(x)$ has a unique root in $\left(0, N^{*}\right)$.

Case 2. $f(x)-x f^{\prime}(x)>0$; namely, $\sigma^{\prime}(x)>0$. Let

$$
\begin{aligned}
F_{1}(x) & =\frac{f(x)}{x} F(x) \\
& =\beta(A-x)+B \frac{f(x)}{x}-\left(\mu+\gamma_{1}+\gamma_{2}+\theta\right) f(x) \\
& =0 .
\end{aligned}
$$

Then,

$$
\begin{aligned}
F_{1}\left(0^{+}\right)= & \lim _{x \rightarrow 0^{+}} F_{1}(x)=+\infty>0, \\
F_{1}(A)= & B \frac{f(A)}{A}-\left(\mu+\gamma_{1}+\gamma_{2}+\theta\right) f(A)<0, \\
F_{1}^{\prime}(x)= & -\beta+B \frac{x f^{\prime}(x)-f(x)}{x^{2}} \\
& -\left(\mu+\gamma_{1}+\gamma_{2}+\theta\right) f^{\prime}(x)<0 .
\end{aligned}
$$

Thus, $F(x)$ has a unique root in $\left(0, N^{*}\right)$. The claimed result follows by the above discussions.

Remark 2. It follows from the above proof that $0<I^{*}<A$.

\subsection{Global Stability}

Theorem 3. $I^{*}$ is globally asymptotically stable with respect to $\Omega$.
Proof. Consider the Lyapunov function

$$
V=\int_{I}^{I^{*}} \frac{u-I^{*}}{u} d u
$$

Then,

$$
\begin{aligned}
\left.\dot{V}\right|_{(3)} & =\frac{I-I^{*}}{I}\left[\sigma(I)(A-I)-\left(\mu+\gamma_{1}+\gamma_{2}+\theta\right) I+B\right] \\
& =\frac{I-I^{*}}{I}\left[\sigma(I)(A-I)-\sigma\left(I^{*}\right)\left(A-I^{*}\right)\right. \\
& \left.-\left(\mu+\gamma_{1}+\gamma_{2}+\theta\right)\left(I-I^{*}\right)\right] \\
& =\frac{I-I^{*}}{I}\left[\left(A-I^{*}\right)\left(\sigma(I)-\sigma\left(I^{*}\right)\right)-\sigma(I)\left(I-I^{*}\right)\right. \\
& \left.-\left(\mu+\gamma_{1}+\gamma_{2}+\theta\right)\left(I-I^{*}\right)\right] \\
& =\frac{\left(I-I^{*}\right)^{2}}{I}\left[\left(A-I^{*}\right) \frac{\sigma(I)-\sigma\left(I^{*}\right)}{I-I^{*}}-\sigma(I)\right. \\
& \left.-\left(\mu+\gamma_{1}+\gamma_{2}+\theta\right)\right] .
\end{aligned}
$$

Here, we proceed by treating two cases.

Case 1. If $\sigma^{\prime}(I) \leq 0$, namely, $f(I)-I f^{\prime}(I) \leq 0$, then it follows from the Lagrange mean value theorem that $(\sigma(I)-$ $\left.\sigma\left(I^{*}\right)\right) /\left(I-I^{*}\right)=\sigma^{\prime}(\xi) \leq 0, \xi \in\left(I, I^{*}\right)$, or $\xi \in\left(I^{*}, I\right)$. As $A-I^{*}=N^{*}-E^{*}-I^{*}>0,\left.\dot{V}\right|_{(3)} \leq 0$ and $\left.\dot{V}\right|_{(3)}=0$ if and only if $I=I^{*}$. Thus, the claimed result follows from LaSalle's Invariance Principle [30].

Case 2. If $\sigma^{\prime}(I)>0$, namely, $f(I)-I f^{\prime}(I)>0$, let

$$
\begin{aligned}
H(I)= & \left(A-I^{*}\right) \frac{\sigma(I)-\sigma\left(I^{*}\right)}{I-I^{*}}-\sigma(I) \\
& -\left(\mu+\gamma_{1}+\gamma_{2}+\theta\right) .
\end{aligned}
$$

Then,

$$
\begin{aligned}
H(0)= & \left(A-I^{*}\right) \frac{\sigma\left(I^{*}\right)}{I^{*}}-\left(\mu+\gamma_{1}+\gamma_{2}+\theta\right)=-\frac{B}{I^{*}} \\
< & 0, \\
H^{\prime}(I)= & \left(A-I^{*}\right) \frac{\sigma^{\prime}(I)\left(I-I^{*}\right)-\left[\sigma(I)-\sigma\left(I^{*}\right)\right]}{\left(I-I^{*}\right)^{2}} \\
& -\sigma^{\prime}(I) .
\end{aligned}
$$

Next, we need to further distinguish two subcases.

Subcase 2.1. If $I>I^{*}$, it follows from the Lagrange mean value theorem that $\sigma(I)-\sigma\left(I^{*}\right)=\sigma^{\prime}(\zeta)\left(I-I^{*}\right), \zeta \in\left(I^{*}, I\right)$. As $f^{\prime \prime}<0, \sigma^{\prime \prime}<0$. Then, for $\epsilon \in(\zeta, I)$,

$$
\begin{aligned}
H^{\prime}(I) & =\left(A-I^{*}\right) \frac{\sigma^{\prime}(I)-\sigma^{\prime}(\zeta)}{I-I^{*}}-\sigma^{\prime}(I) \\
& =\left(A-I^{*}\right) \frac{\sigma^{\prime \prime}(\epsilon)(I-\zeta)}{I-I^{*}}-\sigma^{\prime}(I)<0 .
\end{aligned}
$$




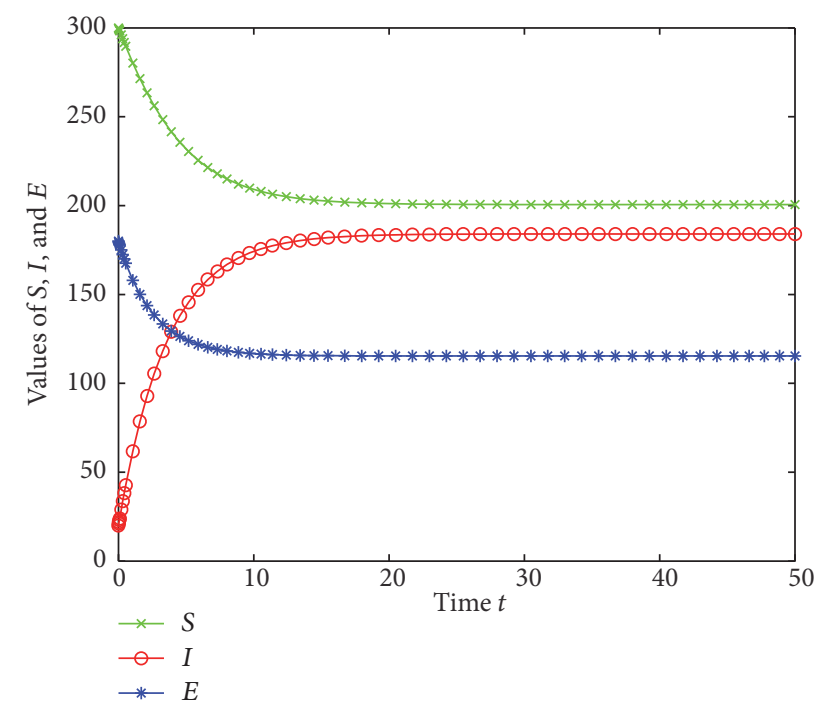

Figure 2: Time plots of $S(t), I(t)$, and $E(t)$ for system (1) given in Example 4.

Subcase 2.2. If $I \leq I^{*}$, it follows from the Lagrange mean value theorem that $\sigma(I)-\sigma\left(I^{*}\right)=\sigma^{\prime}(\varepsilon)\left(I-I^{*}\right), \varepsilon \in\left(I, I^{*}\right)$. As $f^{\prime \prime}<0, \sigma^{\prime \prime}<0$. Then, for $\varsigma \in(I, \varepsilon)$,

$$
\begin{aligned}
H^{\prime}(I) & =\left(A-I^{*}\right) \frac{\sigma^{\prime}(I)-\sigma^{\prime}(\varepsilon)}{I-I^{*}}-\sigma^{\prime}(I) \\
& =\left(A-I^{*}\right) \frac{\sigma^{\prime \prime}(\varsigma)(I-\varepsilon)}{I-I^{*}}-\sigma^{\prime}(I)<0 .
\end{aligned}
$$

It follows from (12)-(13) that $H(I) \leq H(0)<0$. Thus, $\left.\dot{V}\right|_{(3)} \leq 0$. Furthermore, $\left.\dot{V}\right|_{(3)}=0$ if and only if $I=I^{*}$. The claimed result also follows from LaSalle's Invariance Principle [30]. The proof is complete.

Example 4. Consider system (1) with $\delta=5, \mu=0.01$, $\eta_{1}=0.1, \eta_{2}=0.2, \gamma_{1}=0.08, \gamma_{2}=0.08895, \theta=0.01$, and $\sigma(I)=0.04487 I /(1+\sqrt{I})$. Figure 2 displays the time plot of this system with initial condition $(S(0), I(0), E(0))=$ $(300,20,180)$. From Figure 2 , the values of $S(t), I(t)$, and $E(t)$ tend to a constant, respectively. Furthermore, $I(t)$ tends to a positive constant. This shows that $I^{*}$ is globally asymptotically stable.

Remark 5. Theorem 3 reveals that $I(t)$ tends to a positive constant $I^{*}$. From an epidemiological standpoint, it indicates that computer virus would persist in network. Thus, one can conclude that any effort in eradicating computer virus is doomed to failure. Thus, the best achievable goal is to make the number of infected computers below an acceptable level (i.e., as low as possible). Note that $I^{*}$ cannot be expressed in a specific formula, and it follows from Remark 2 that $0<I^{*}<$ $A$. Then, one could keep the value of $A$ below an acceptable threshold. To this end, the following result is made.

Theorem 6. From Equation (3), $A=\delta\left(\eta_{1}+\eta_{2}\right) / \mu\left(\mu+\gamma_{1}+\eta_{1}+\right.$ $\left.\eta_{2}\right)$. Then $\partial A / \partial \mu<0, \partial A / \partial \gamma_{1}<0, \partial A / \partial \delta>0, \partial A / \partial \eta_{1}>0$, and $\partial A / \partial \eta_{2}>0$.

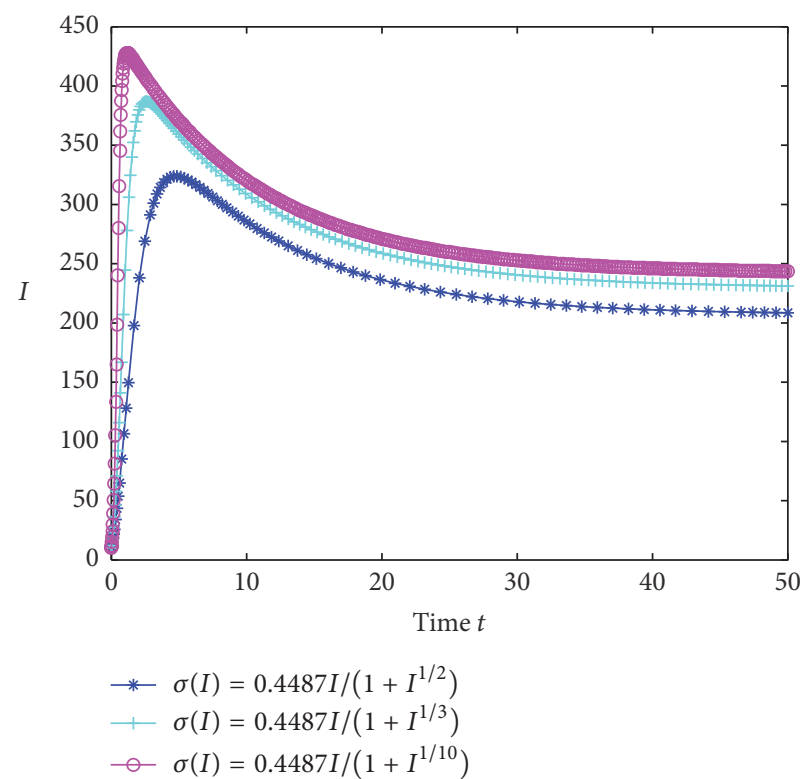

FIGURE 3: An illustration of the impact of $\sigma(I)$ on $I(t)$ for system (1) given in Example 8.

Proof. Since $A=\delta\left(\eta_{1}+\eta_{2}\right) / \mu\left(\mu+\gamma_{1}+\eta_{1}+\eta_{2}\right)$, then

$$
\begin{aligned}
\frac{\partial A}{\partial \mu} & =-\frac{\delta\left(\eta_{1}+\eta_{2}\right)\left(2 \mu+\gamma_{1}+\eta_{1}+\eta_{2}\right)}{\mu^{2}\left(\mu+\gamma_{1}+\eta_{1}+\eta_{2}\right)^{2}}<0, \\
\frac{\partial A}{\partial \gamma_{1}} & =-\frac{\delta\left(\eta_{1}+\eta_{2}\right)}{\mu\left(\mu+\gamma_{1}+\eta_{1}+\eta_{2}\right)^{2}}<0, \\
\frac{\partial A}{\partial \delta} & =\frac{\eta_{1}+\eta_{2}}{\mu\left(\mu+\gamma_{1}+\eta_{1}+\eta_{2}\right)}>0, \\
\frac{\partial A}{\partial \eta_{1}} & =\frac{\delta\left(\mu+\gamma_{1}\right)}{\mu\left(\mu+\gamma_{1}+\eta_{1}+\eta_{2}\right)^{2}}>0, \\
\frac{\partial A}{\partial \eta_{2}} & =\frac{\delta\left(\mu+\gamma_{1}\right)}{\mu\left(\mu+\gamma_{1}+\eta_{1}+\eta_{2}\right)^{2}}>0 .
\end{aligned}
$$

Thus, the proof is complete.

Remark 7. Theorem 6 implies the effects of some system parameters on the value of $A$.

In addition, the following two examples exhibit the impacts of $\sigma(I)$ and $\theta$ on $I$, respectively.

Example 8. Consider system (1) with $\delta=5, \mu=0.01$, $\eta_{1}=0.01, \eta_{2}=0.04, \gamma_{1}=0.04, \gamma_{2}=0.08895$, and $\theta=0.01$. Figure 3 demonstrates the time plot of this system with initial condition $(S(0), I(0), E(0))=(450,10,40)$.

Example 9. Consider system (1) with $\delta=5, \mu=0.01$, $\eta_{1}=0.04, \eta_{2}=0.08, \gamma_{1}=0.08, \gamma_{2}=0.08895$, and $\sigma(I)=0.004487 I /(1+\sqrt{I})$. Figure 4 shows the time plot of this system with initial condition $(S(0), I(0), E(0))=(450,10,40)$. 


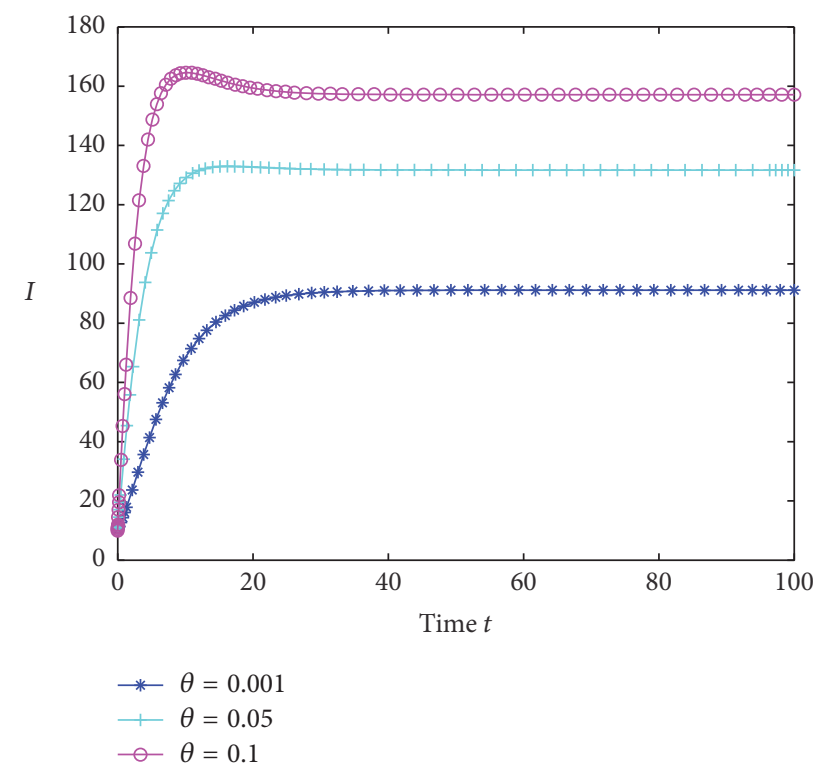

FIGURE 4: An illustration of the impact of $\theta$ on $I(t)$ for system (1) given in Example 9.

Figures 3 and 4 show the effects of different infection rates and infected removable media on virus diffusion, respectively.

\section{Optimal Control of the Model}

To make a tradeoff between control cost and control effect, the control variable $u(t)$ meaning the control strategy is applied in the proposed model (system (1)). Then, one can get the controlled dynamical system

$$
\begin{aligned}
& \dot{S}=\gamma_{2} I+\eta_{2} E-\mu S-\sigma(I) S-\gamma_{1} S-\theta S, \\
& \dot{I}=\sigma(I) S+\theta S-\mu I-\gamma_{1} I-u(t) I+\eta_{1} E, \\
& \dot{E}=\delta+\gamma_{1} S+\gamma_{1} I-\mu E-\eta_{1} E-\eta_{2} E,
\end{aligned}
$$

with initial condition $(S(0), I(0), E(0))^{T} \in \Omega^{\prime}$, where

$$
\Omega^{\prime}=\left\{(S, I, E)^{T} \in R_{+}^{3} \mid S+I+E \leq \frac{\delta}{\mu}\right\} .
$$

The admissible control set is

$$
U=\left\{u(\cdot) \in L^{2}[0, T] \mid \underline{\gamma_{2}} \leq u(\cdot) \leq \bar{\gamma}_{2}\right\},
$$

where $\gamma_{2}$ and $\bar{\gamma}_{2}$ are positive constants and $0<\underline{\gamma}_{2}<\bar{\gamma}_{2}<1$.

Let $\mathbf{x}(t)=(S(t), I(t), E(t))^{T}$. Then system (15) can be written in matrix notation as

$$
\frac{d \mathbf{x}(t)}{d t}=\mathbf{f}(\mathbf{x}(t), u(t)), \quad 0 \leq t \leq T,
$$

with initial condition $\mathbf{x}(0) \in \Omega^{\prime}$.

Now, the objective is to find a control variable $u(\cdot)$ so as to minimize both the number of infected computers and the total budget for treatment and vaccination during the time period $[0, T]$. That is, it suffices to solve the optimal control problem.

$$
\text { Minimize }_{u(\cdot) \in U} \quad J(u(\cdot))=\int_{0}^{T} L(\mathbf{x}(t), u(t)) d t
$$

subject to system (18), where

$$
L(\mathbf{x}, u)=I+\frac{\epsilon u^{2}}{2}
$$

is the Lagrangian and $\epsilon>0$ is a tradeoff factor based on the control cost and control effect.

Theorem 10. The optimal control problem (19) has an optimal control.

Proof. From equations (19) and (20), one can get that

(1) there exist $u(\cdot) \in U$ such that system (18) is solvable,

(2) the admissible control set $U$ is convex and closed,

(3) the right-hand side of system (18) is bounded by a linear function in $\mathbf{x}$,

(4) $L(\mathbf{x}, u)$ is convex on $U$,

(5) there exist $\rho=2, c_{1}=\epsilon / 2$, and $c_{2}=0$ such that $L(\mathbf{x}, u) \geq(\epsilon / 2)\|u\|_{2}^{2}$.

Thus, the claimed result follows directly from [31].

Theorem 11. Suppose $\widetilde{u}(\cdot)$ is an optimal control for the optimal control problem (19), and $(\widetilde{S}(t), \widetilde{I}(t), \widetilde{E}(t))^{T}$ is the solution to system (18) with $u(\cdot)=\widetilde{u}(\cdot)$. Then, there exist functions $\widetilde{\lambda_{1}}(t)$, $\widetilde{\lambda_{2}}(t)$, and $\widetilde{\lambda_{3}}(t), 0 \leq t \leq T$, such that

$$
\begin{aligned}
& \frac{d \widetilde{\lambda_{1}}(t)}{d t}= \widetilde{\lambda_{1}}(t)\left(\mu+\gamma_{1}+\theta+\sigma(\widetilde{I}(t))\right)-\gamma_{1} \widetilde{\lambda_{3}}(t) \\
&-\widetilde{\lambda_{2}}(t)(\theta+\sigma(\widetilde{I}(t))), \\
& \frac{d \widetilde{\lambda_{2}}(t)}{d t}=-1-\widetilde{\lambda_{1}}(t)(\mu-C \widetilde{S}(t))-\gamma_{1} \widetilde{\lambda_{3}}(t) \\
&+\widetilde{\lambda_{2}}(t)\left(\mu+\gamma_{1}+\widetilde{u}(t)-C \widetilde{S}(t)\right), \\
& \frac{d \widetilde{\lambda_{3}}(t)}{d t}=-\eta_{2} \widetilde{\lambda_{1}}(t)-\eta_{1} \widetilde{\lambda_{2}}(t)+\left(\mu+\eta_{1}+\eta_{2}\right) \widetilde{\lambda_{3}}(t), \\
& \quad 0 \leq t \leq T,
\end{aligned}
$$

with transversality conditions $\widetilde{\lambda_{1}}(T)=\widetilde{\lambda_{2}}(T)=\widetilde{\lambda_{3}}(T)=0$, where

$$
C=\frac{\beta f(\widetilde{I}(t))-\beta \widetilde{I}(t) f^{\prime}(\widetilde{I}(t))}{f^{2}(\widetilde{I}(t))} .
$$


Furthermore, we have

$$
\begin{array}{r}
u^{*}(t) \\
=\max \left\{\min \left\{\frac{\left(\widetilde{\lambda_{2}}(t)-\widetilde{\lambda_{1}}(t)\right) \widetilde{I}(t)}{\epsilon}, \bar{\gamma}_{2}\right\}, \underline{\gamma_{2}}\right\}, \\
0 \leq t \leq T .
\end{array}
$$

Proof. Note that the Hamiltonian is

$$
\begin{aligned}
& H\left(S, I, E, \lambda_{1}, \lambda_{2}, \lambda_{3}, u\right) \\
& \quad=I+\frac{\epsilon}{2} u^{2}+\lambda_{1} \frac{d S}{d t}+\lambda_{2} \frac{d I}{d t}+\lambda_{3} \frac{d E}{d t},
\end{aligned}
$$

where $\lambda_{1}, \lambda_{2}$, and $\lambda_{3}$ are undetermined.

Then, applying the Pontryagin Minimum Principle [32], there exist functions $\widetilde{\lambda_{1}}(t), \widetilde{\lambda_{2}}(t)$, and $\widetilde{\lambda_{3}}(t), 0 \leq t \leq T$, such that

$$
\begin{aligned}
& \frac{d \widetilde{\lambda_{1}}(t)}{d t} \\
& =-\frac{\partial H\left(\widetilde{S}(t), \widetilde{I}(t), \widetilde{E}(t), \widetilde{\lambda_{1}}(t), \widetilde{\lambda_{2}}(t), \widetilde{\lambda_{3}}(t), \widetilde{u}(t)\right)}{\partial S} \\
& \frac{d \widetilde{\lambda_{2}}(t)}{d t} \\
& =-\frac{\partial H\left(\widetilde{S}(t), \widetilde{I}(t), \widetilde{E}(t), \widetilde{\lambda_{1}}(t), \widetilde{\lambda_{2}}(t), \widetilde{\lambda_{3}}(t), \widetilde{u}(t)\right)}{\partial I}, \\
& \frac{d \widetilde{\lambda_{3}}(t)}{d t} \quad 0 \leq t \leq T . \\
& =-\frac{\partial H\left(\widetilde{S}(t), \widetilde{I}(t), \widetilde{E}(t), \widetilde{\lambda_{1}}(t), \widetilde{\lambda_{2}}(t), \widetilde{\lambda_{3}}(t), \widetilde{u}(t)\right)}{\partial E \quad},
\end{aligned}
$$

Hence, system (21) follows by a straightforward calculation. As the terminal cost is unspecified and the final state is free, the transversality conditions hold.

Note that the optimality condition $H(\widetilde{S}(\cdot), \widetilde{I}(\cdot), \widetilde{E}(\cdot)$, $\left.\widetilde{\lambda_{1}}(\cdot), \widetilde{\lambda_{2}}(\cdot), \widetilde{\lambda_{3}}(\cdot), \widetilde{u}(\cdot)\right)=\min _{u(\cdot) \in U} H\left(\widetilde{S}(\cdot), \widetilde{I}(\cdot), \widetilde{E}(\cdot), \widetilde{\lambda_{1}}(\cdot)\right.$, $\left.\widetilde{\lambda_{2}}(\cdot), \widetilde{\lambda_{3}}(\cdot), u(\cdot)\right)$. Then, either

$$
\begin{gathered}
\frac{\partial H\left(\widetilde{S}(t), \widetilde{I}(t), \widetilde{E}(t), \widetilde{\lambda_{1}}(t), \widetilde{\lambda_{2}}(t), \widetilde{\lambda_{3}}(t), \widetilde{u}(t)\right)}{\partial u} \\
=\epsilon \widetilde{u}(t)+\widetilde{\lambda_{1}}(t) \widetilde{I}(t)-\widetilde{\lambda_{2}}(t) \widetilde{I}(t)=0
\end{gathered}
$$

or $\widetilde{u}(t)=\underline{\gamma_{2}}$ or $\widetilde{u}(t)=\bar{\gamma}_{2}$. Thus, the proof is complete.
By combining the above discussions, the optimality system for the optimal control problem (19) can be derived as follows:

$$
\begin{aligned}
& \frac{d S(t)}{d t} \\
& =u(t) I(t)+\eta_{2} E(t)-\mu S(t)-\sigma(I(t)) S(t) \\
& \quad-\gamma_{1} S(t)-\theta S(t),
\end{aligned}
$$

$\frac{d I(t)}{d t}$

$$
\begin{aligned}
= & \sigma(I(t)) S(t)+\theta S(t)-\mu I(t)-\gamma_{1} I(t) \\
& -u(t) I(t)+\eta_{1} E(t),
\end{aligned}
$$

$\frac{d E(t)}{d t}$

$$
=\delta+\gamma_{1} S(t)+\gamma_{1} I(t)-\mu E(t)-\eta_{1} E(t)-\eta_{2} E(t),
$$

$\frac{d \lambda_{1}(t)}{d t}$

$$
\begin{aligned}
= & \lambda_{1}(t)\left(\mu+\gamma_{1}+\theta+\sigma(I(t))\right) \\
& -\lambda_{2}(t)(\theta+\sigma(I(t)))-\gamma_{1} \lambda_{3}(t),
\end{aligned}
$$

$\frac{d \lambda_{2}(t)}{d t}$

$$
\begin{array}{r}
=-1-\lambda_{1}(t)(\mu-C S(t)) \\
+\lambda_{2}(t)\left(\mu+\gamma_{1}+u(t)-C S(t)\right)-\gamma_{1} \lambda_{3}(t), \\
\frac{d \lambda_{3}(t)}{d t}=-\eta_{2} \lambda_{1}(t)-\eta_{1} \lambda_{2}(t)+\left(\mu+\eta_{1}+\eta_{2}\right) \lambda_{3}(t), \\
u(t)=\max \left\{\min \left\{\frac{\left(\lambda_{2}(t)-\lambda_{1}(t)\right) I(t)}{\epsilon}, \bar{\gamma}_{2}\right\}, \underline{\gamma_{2}}\right\}, \\
0 \leq t \leq T,
\end{array}
$$

with $(S(0), I(0), E(0))^{T} \in \Omega^{\prime}$, and $\lambda_{1}(T)=\lambda_{2}(T)=\lambda_{3}(T)=$ 0 .

Next, the effectiveness of optimal control will be examined. Here we have to point out that all parameter values are chosen hypothetically due to the unavailability of real-world data.

Example 12. Suppose that $\delta=2, \mu=0.01, \eta_{1}=0.012, \eta_{2}=$ $0.021, \gamma_{1}=0.01, \theta=0.015, \underline{\gamma_{2}}=0.001, \bar{\gamma}_{2}=0.8, \sigma(I)=$ $0.005 I /(1+\sqrt{I}), \epsilon=15$, and $T=100$. The optimality system (18) with $(S(0), I(0), E(0))=(100,60,40)$ is to be numerically solved with the backward-forward Runge-Kutta fourth-order scheme. Then Figures 5-7 and Table 1 are obtained.

Figures 5 and 6 exhibit the evolution of $S$ and $I$ with different control strategies, respectively. From these two figures, it is natural to see that the optimal control $\widetilde{u}$ is superior to others. 
TABLE 1: The values of $I$ and objective function $J$ under different control strategies $u$.

\begin{tabular}{ccccccc}
\hline & $u=\widetilde{u}$ & $u=0.001$ & $u=0.2$ & $u=0.4$ & \multicolumn{1}{c}{$u=0.6$} & 6.1 \\
\hline$I(u)$ & 5.5 & 104.1 & 19.9 & 9.5 & 4.3 \\
$J(u)$ & 748.1 & 9894.8 & 2637.1 & 1671.4 & 1373.1 & 1322.9 \\
\hline
\end{tabular}

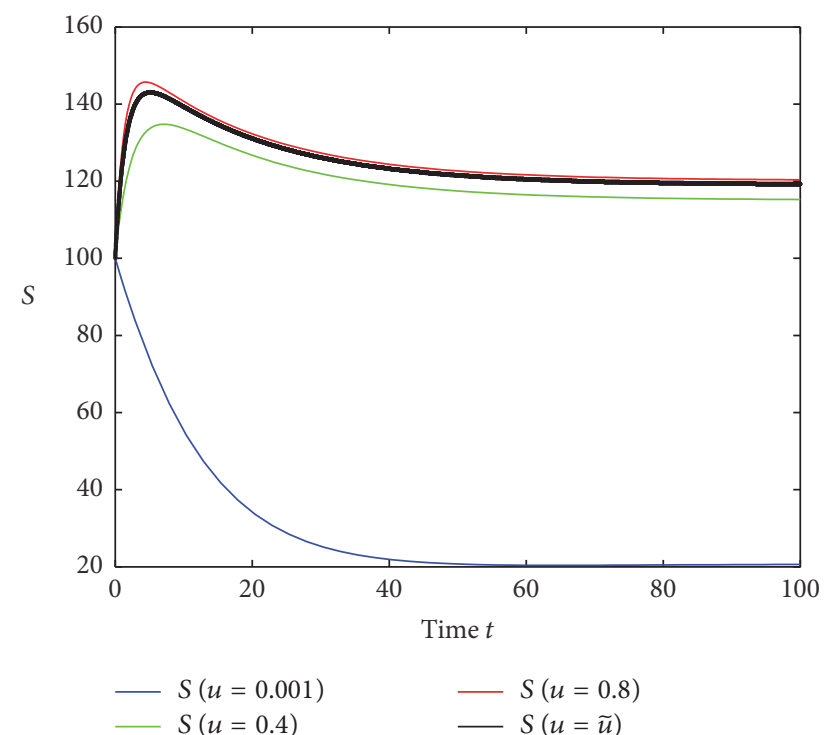

FIGURE 5: Evolution of number of susceptible computers with different control strategies.

Figure 7 shows the corresponding optimal control strategy.

Table 1 lists the values of infected computers $I$ and objective function $J$ under different control strategies. It is easy to conclude that $\tilde{u}$ is the best choice.

\section{Conclusions and Prospects}

This paper has investigated an SIES model with generic nonlinear infection rate. A thorough analysis shows that the unique (viral) equilibrium is globally asymptotically stable. This result implies that any effort in eradicating computer virus is inoperative. As a result, a countermeasure, which mainly aims to maintain the number of infected computers at an acceptable level, and optimal control analysis of the proposed model have been posed. Some numerical examples are also included.

The study of this model not only implies some new practical measures but also gives a theoretical support to the usefulness of some existing antivirus strategies and provides the basis to developing many other more elaborated models.

The study can be continued in several directions. First of all, it would be interesting for complex network (e.g., scale-free network) because our model is a homogeneous model. Next, delays (or pulses) could be incorporated in our model so as to characterize the delay in the development of new vaccine (or the emergency of new virus). Finally,

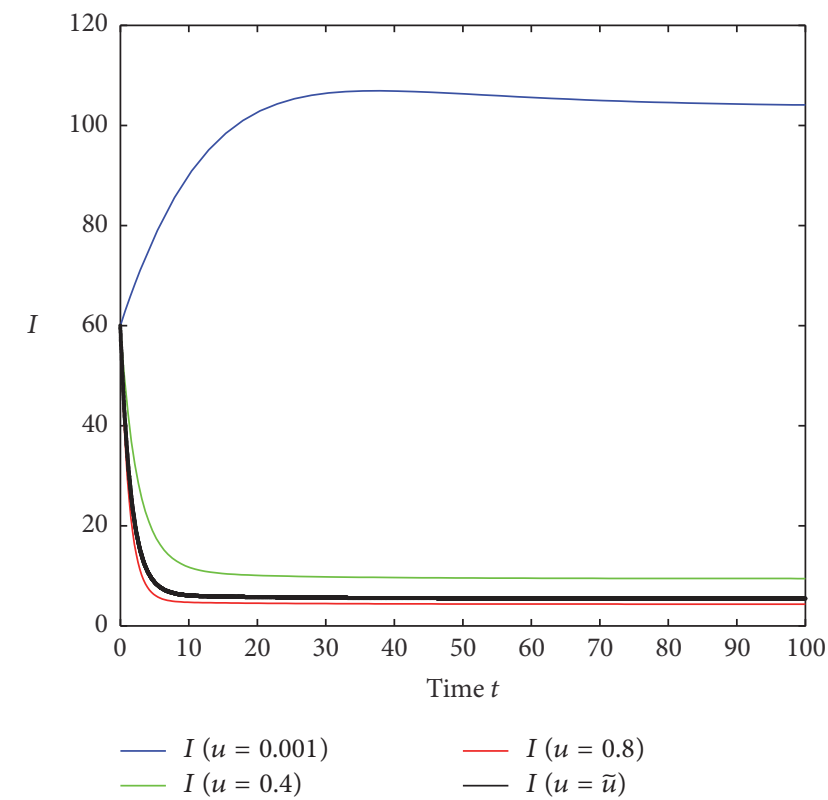

FIGURE 6: Evolution of number of infected computers with different control strategies.

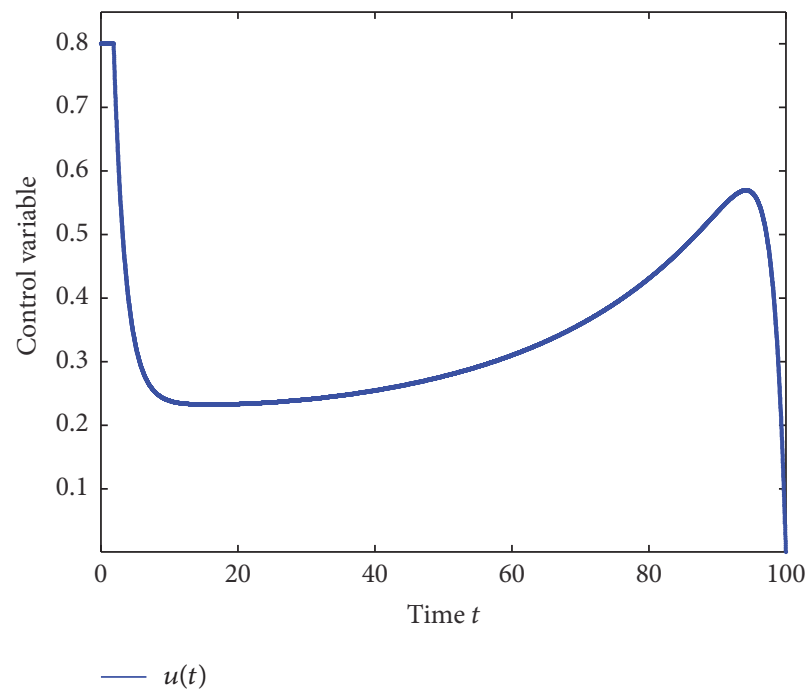

Figure 7: An optimal control function for system (18).

our model involves a relatively large number of parameters, which are very difficult to establish with accuracy. Therefore, it is appropriate to modify our model by considering the parameters to be random variables. 


\section{Competing Interests}

The authors declare that there are no competing interests regarding the publication of this paper.

\section{Acknowledgments}

This work is supported by Natural Science Foundation of China (Grant nos. 11547148, 61603065, and 61503307), Innovation Project of the Common Key Technology of Chongqing Science and Technology Industry (Grant no. cstc2015zdcyztzx40008), Scientific and Technological Research Program of Chongqing Municipal Education Commission (Grant nos. KJ1500415 and KJ1500904), and Doctoral Scientific Research Foundation of Chongqing University of Posts and Telecommunications (Grant nos. A2015-02 and A2016-10).

\section{References}

[1] Z.-h. Zuo, Q.-x. Zhu, and M.-t. Zhou, "On the time complexity of computer viruses," Institute of Electrical and Electronics Engineers. Transactions on Information Theory, vol. 51, no. 8, pp. 2962-2966, 2005.

[2] J. O. Kephart and S. R. White, "Directed-graph epidemiological models of computer viruses," in Proceedings of the IEEE Computer Society Symposium on Research in Security and Privacy, pp. 343-358, May 1991.

[3] J. O. Kephart and S. R. White, "Measuring and modeling computer virus prevalence," in Proceedings of the 1993 IEEE Computer Society Symposium on Research in Security and Privacy, Oakland, Calif, USA, May 1993.

[4] L. Feng, X. Liao, H. Li, and Q. Han, "Hopf bifurcation analysis of a delayed viral infection model in computer networks," Mathematical and Computer Modelling, vol. 56, no. 7-8, pp. 167179, 2012.

[5] C. Gan, X. Yang, W. Liu, Q. Zhu, and X. Zhang, "Propagation of computer virus under human intervention: a dynamical model," Discrete Dynamics in Nature and Society, vol. 2012, Article ID 106950, 8 pages, 2012.

[6] Q. Zhu, X. Yang, and J. Ren, "Modeling and analysis of the spread of computer virus," Communications in Nonlinear Science and Numerical Simulation, vol. 17, no. 12, pp. 5117-5124, 2012.

[7] C. Gan, X. Yang, W. Liu, and Q. Zhu, "A propagation model of computer virus with nonlinear vaccination probability," Communications in Nonlinear Science and Numerical Simulation, vol. 19, no. 1, pp. 92-100, 2014.

[8] J. Amador, "The stochastic SIRA model for computer viruses," Applied Mathematics and Computation, vol. 232, pp. 1112-1124, 2014.

[9] J. Ren and Y. Xu, "Stability and bifurcation of a computer virus propagation model with delay and incomplete antivirus ability," Mathematical Problems in Engineering, vol. 2014, Article ID 475934, 9 pages, 2014.

[10] B. K. Mishra and S. K. Pandey, "Dynamic model of worm propagation in computer network," Applied Mathematical Modelling, vol. 38, no. 7-8, pp. 2173-2179, 2014.

[11] Y. Yao, X. Feng, W. Yang, W. Xiang, and F. Gao, "Analysis of a delayed Internet worm propagation model with impulsive quarantine strategy," Mathematical Problems in Engineering, vol. 2014, Article ID 369360, 18 pages, 2014.
[12] C. Gan, "Modeling and analysis of the effect of network eigenvalue on viral spread," Nonlinear Dynamics, vol. 84, no. 3, pp. 1727-1733, 2016.

[13] W. Liu, C. Liu, Z. Yang, X. Liu, Y. Zhang, and Z. Wei, "Modeling the propagation of mobile malware on complex networks," Communications in Nonlinear Science and Numerical Simulation, vol. 37, pp. 249-264, 2016.

[14] C. Zhang and H. Huang, "Optimal control strategy for a novel computer virus propagation model on scale-free networks," Physica A. Statistical Mechanics and its Applications, vol. 451, pp. 251-265, 2016.

[15] B. K. Mishra, K. Haldar, and D. N. Sinha, "Impact of information based classification on network epidemics," Scientific Reports, vol. 6, Article ID 28289, 2016.

[16] J. R. C. Piqueira, A. A. de Vasconcelos, C. E. C. J. Gabriel, and V. O. Araujo, "Dynamic models for computer viruses," Computers and Security, vol. 27, no. 7-8, pp. 355-359, 2008.

[17] J. R. C. Piqueira and V. O. Araujo, "A modified epidemiological model for computer viruses," Applied Mathematics and Computation, vol. 213, no. 2, pp. 355-360, 2009.

[18] X. Han and Q. Tan, "Dynamical behavior of computer virus on internet," Applied Mathematics and Computation, vol. 217, no. 6, pp. 2520-2526, 2010.

[19] B. K. Mishra and S. K. Pandey, "Dynamic model of worms with vertical transmission in computer network," Applied Mathematics and Computation, vol. 217, no. 21, pp. 8438-8446, 2011.

[20] Q. Zhu, X. Yang, L.-X. Yang, and X. Zhang, "A mixing propagation model of computer viruses and countermeasures," Nonlinear Dynamics, vol. 73, no. 3, pp. 1433-1441, 2013.

[21] L.-X. Yang and X. Yang, "The impact of nonlinear infection rate on the spread of computer virus," Nonlinear Dynamics, vol. 82, no. 1-2, pp. 85-95, 2015.

[22] A. Lahrouz, L. Omari, D. Kiouach, and A. Belmaâti, "Complete global stability for an SIRS epidemic model with generalized non-linear incidence and vaccination," Applied Mathematics and Computation, vol. 218, no. 11, pp. 6519-6525, 2012.

[23] C. Gan, X. Yang, W. Liu, Q. Zhu, and X. Zhang, "An epidemic model of computer viruses with vaccination and generalized nonlinear incidence rate," Applied Mathematics and Computation, vol. 222, pp. 265-274, 2013.

[24] L.-X. Yang and X. Yang, "The spread of computer viruses under the influence of removable storage devices," Applied Mathematics and Computation, vol. 219, no. 8, pp. 3914-3922, 2012.

[25] C. Gan, X. Yang, Q. Zhu, J. Jin, and L. He, “The spread of computer virus under the effect of external computers," Nonlinear Dynamics, vol. 73, no. 3, pp. 1615-1620, 2013.

[26] Q. Zhu, X. Yang, L.-X. Yang, and C. Zhang, "Optimal control of computer virus under a delayed model," Applied Mathematics and Computation, vol. 218, no. 23, pp. 11613-11619, 2012.

[27] L. Chen, K. Hattaf, and J. Sun, "Optimal control of a delayed SLBS computer virus model," Physica A, vol. 427, no. 1, pp. 244250, 2015.

[28] L.-X. Yang, M. Draief, and X. Yang, "The optimal dynamic immunization under a controlled heterogeneous node-based SIRS model," Physica A. Statistical Mechanics and its Applications, vol. 450, pp. 403-415, 2016.

[29] H. R. Thieme, "Asymptotically autonomous differential equations in the plane," The Rocky Mountain Journal of Mathematics, vol. 24, no. 1, pp. 351-380, 1994. 
[30] R. C. Robinson, An Introduction to Dynamical System: Continuous and Discrete, Prentice Hall, New York, NY, USA, 2004.

[31] M. I. Kamien and N. L. Schwartz, Dynamic Optimization: The Calculus of Variations and Optimal Control in Economics and Management, Elsevier Science, Amsterdam, The Netherlands, 2000.

[32] D. Liberzon, Calculus of Variations and Optimal Control Theory: A Concise Introduction, Princeton University Press, Princeton, NJ, USA, 2012. 


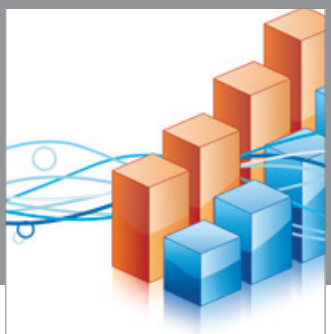

Advances in

Operations Research

vatem alat4

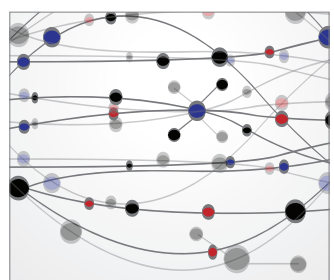

\section{The Scientific} World Journal
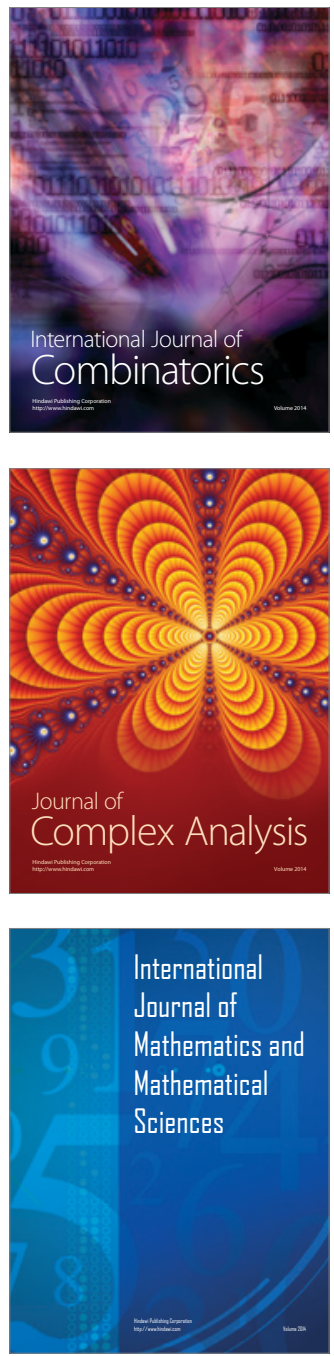
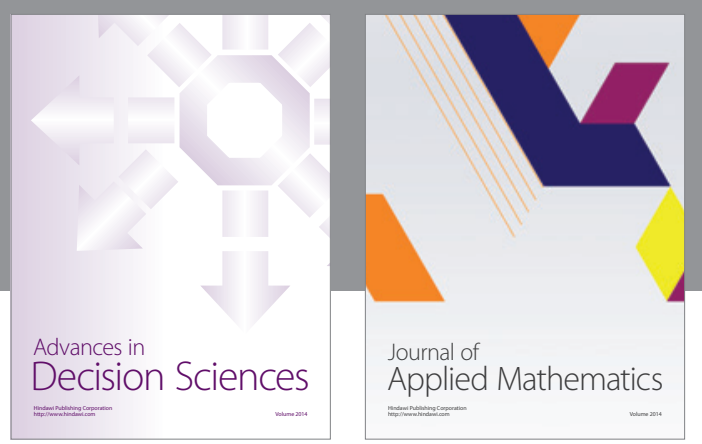

Algebra

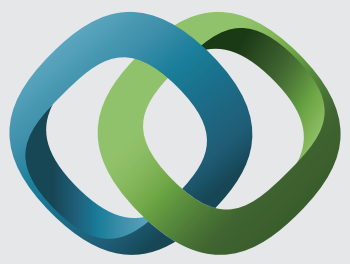

\section{Hindawi}

Submit your manuscripts at

https://www.hindawi.com
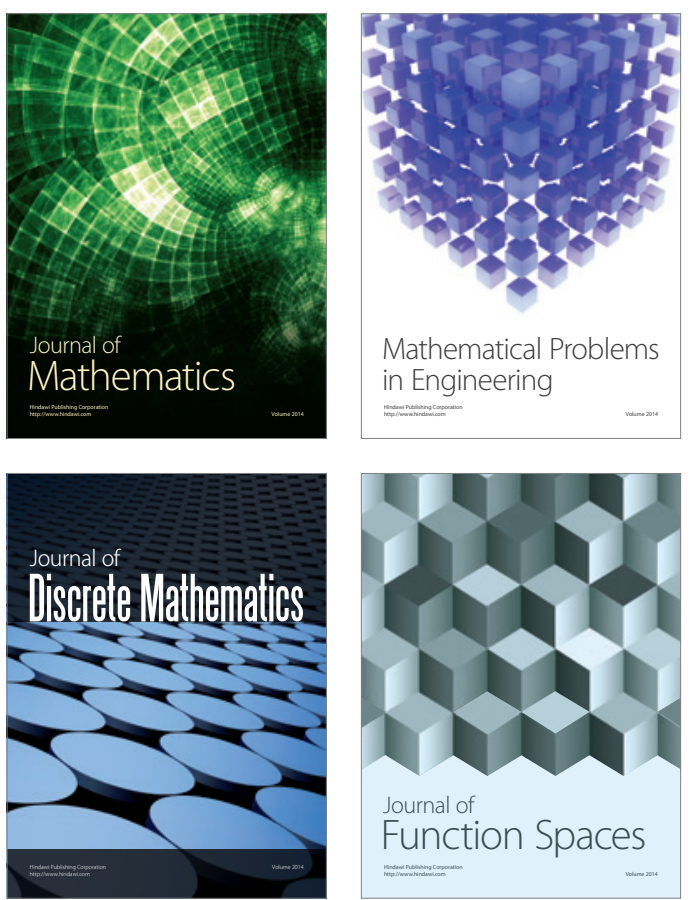

Mathematical Problems in Engineering
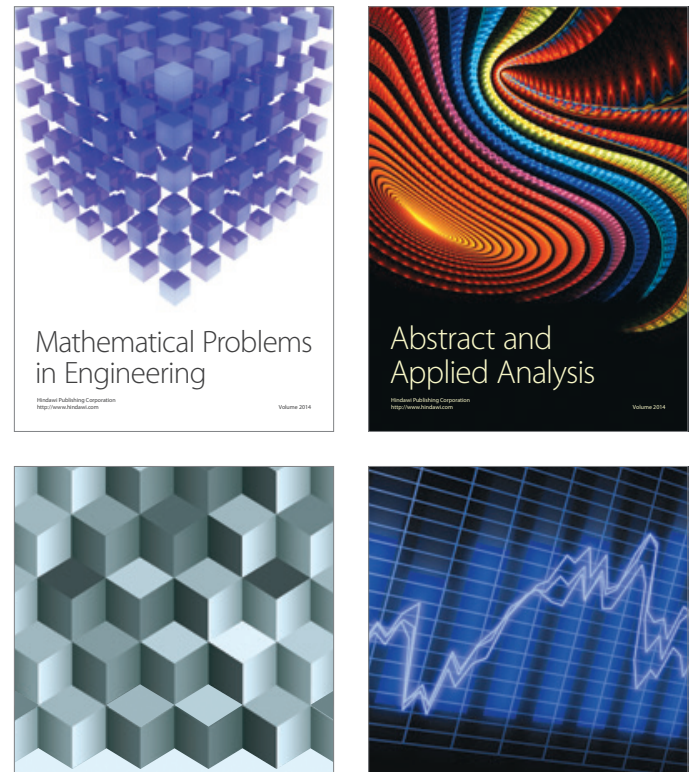

Journal of

Function Spaces

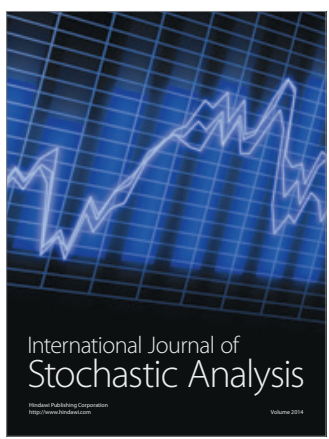

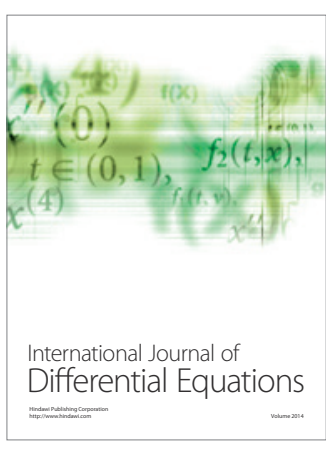
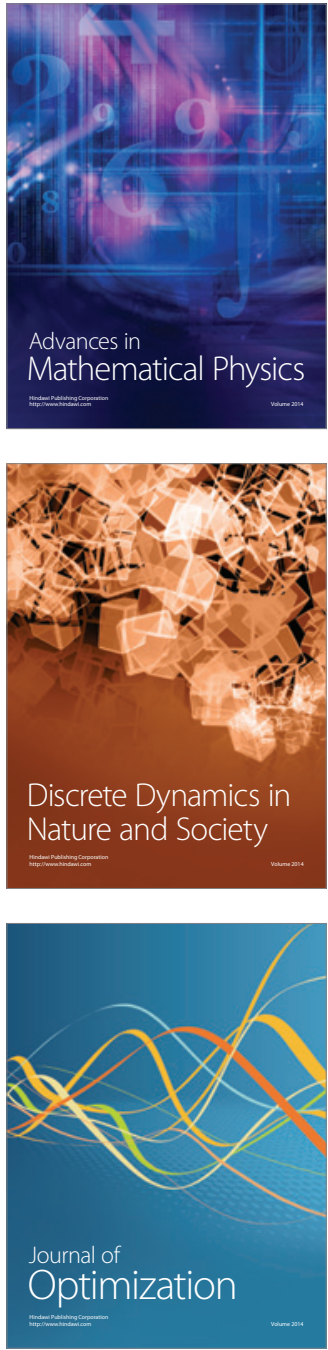